\title{
Performance of TD-CDMA systems during crossed slots
}

\author{
Jad NASREDDINE and Xavier LAGRANGE \\ Multimedia Networks and Services Department, GET / ENST de Bretagne \\ 2 rue de la châtaigneraie, CS 17607, 35576 Cesson Sévigné Cedex, France \\ e-mail: \{jad.nasreddine and xavier.lagrange\}@enst-bretagne.fr
}

\begin{abstract}
Many multimedia services of the next generation mobile telecommunication systems have an asymmetric traffic distribution between uplink and downlink. The combination of the Time Division Duplex (TDD) with the Time Division Multiple Access (TDMA) technique in the Universal Mobile Telecommunications System (UMTS) allows an asymmetric use of radio resources due to the flexible allocation of time slots. To conserve this flexibility, the uplink and downlink slot distribution is prefered to be different in cells due to the different rates of asymmetry. Hence, crossed slots, where a set of cells are active in downlink and another set are active in uplink, must be allowed. In crossed slots, intermobile and inter-base station interferences appear. Intermobile interference is very difficult to measure and may induce very high outage probability when two close mobiles are active in opposite link directions during the same slot. In this paper, a simplified optimum power control is used to study the performance of a TD-CDMA system during a crossed slot. Simulation results has shown that the system performance may be better in crossed slots than in uplink slots for some mobile distributions.
\end{abstract}

\section{BACKGROUND}

In many applications of the next generation wireless systems, most of the traffic load is expected to appear in downlink. To efficiently manage the traffic asymmetry, the resource unit distribution between uplink and downlink must be adequate to the rate of asymmetry in cells.

In the TDD mode of UMTS, uplink and downlink are separated in time domain. Furthermore, the frame is divided into 15 time slots that can be allocated either for uplink or for downlink in a cell. The boundaries between uplink and downlink slots in a cell are called the switching points. Moreover, the same time slot may be used for uplink in some cells and for downlink in other cells. In this case, the time slot is called a crossed slot (figure 1).

To accommodate the slot management to asymmetric traffics, different techniques were proposed. Some of these techniques are based on common switching point where all cells have the same slot configuration. The common switching point technique limits the flexibility of the TD-CDMA system since all cells do not have the same asymmetric ratio. In the diversified switching point technique [1], the number of slots allocated to the downlink and to the uplink in a cell depends on the rate of asymmetry between both links. Hence, the resource units

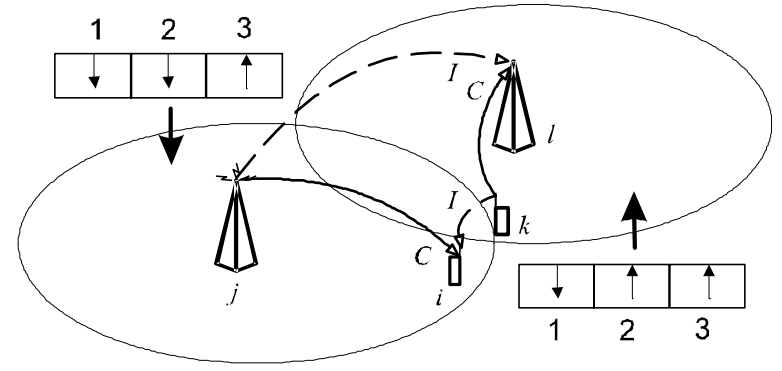

Fig. 1. Crossed time slot (2) in a sytem with two cells.

can be efficiently distributed among both links. When the latter technique is used, the system may experience very annoying types of interference [2] (i.e. mobile-mobile and base station-base station interference (figure 1)). These types of interference appear in crossed slots and may increase significantly the outage probability.

In this paper, the performance of the TD-CDMA system is investigated during crossed slots. The system performance during these slots is compared to the system performance during uplink slots where the intracell interference is higher than in downlink slots. To compare the performance during these types of slot, the simplified optimum power control [3][4] is used to compute the maximum achievable Carrier to Interference Ratio $(C I R)$.

In Section 2, the system model is described. The simplified optimum power control is presented in section 3 . The system performance in crossed slots is compared to the system performance in uplink slots using simulation results in section 4. Conclusions and useful comments are highlighted in the last section.

\section{System MODEL}

The system performance is evaluated in a simulation platform consisting of 25 hexagonal cells. The cell radius is 100 meters. A wraparound technique is applied to ensure that each cell is completely surrounded by a symmetric pattern. All link gains are assumed to be known through intelligent measuring. We suppose that a mobile is always served by the best-received base station and that joint 

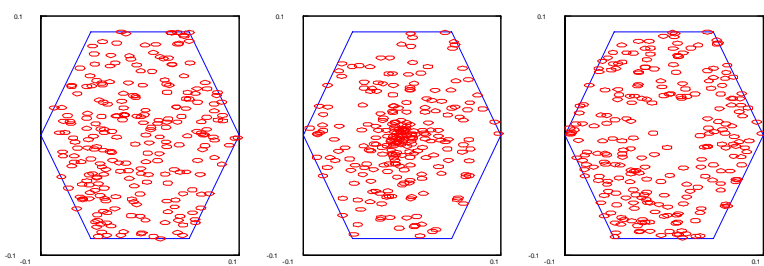

Fig. 2. The three types of mobile distribution

detection technique is used [5]. We denote $\beta_{d}$ and $\beta_{u}$ the factors of remaining intracell interference respectively in downlink and in uplink after the joint detection procedure. Thus, the interference power estimated at the receiver after the joint detection is given by:

$$
I=\beta_{l} . I_{\text {intra }}+I_{\text {inter }}
$$

where $l$ is the link type, $I_{\text {intra }}$ and $I_{\text {inter }}$ are the intracell and intercell interference powers. The values of $\beta_{d}$ and $\beta_{u}$ are respectively 0.1 and 0.2 . We assume that the interference power is much larger than the white noise in the receiver, therefore, the white noise is not considered in our study.

If a base station $j$ is transmitting during a time slot $n$, the corresponding cell is called downlink-cell. If the mobiles of a cell $j$ are transmitting during a time slot $n$, the cell $j$ is called uplink-cell. In crossed slots, uplink and downlink-cells are randomly distributed with an asymmetry rate $a_{r}=C_{u} /\left(C_{d}+C_{d}\right)$, where $C_{u}$ and $C_{d}$ are the number of uplink and downlink-cells respectively. We also assume that all mobiles request the same type of services. An Okumura-hata-cost231 model with shadowing is used [6][7]. A minimum distance of $0.2 \mathrm{~m}$ between mobiles and $1 \mathrm{~m}$ between mobiles and base stations are considered.

Three types of mobile distribution are used. In the first distribution, mobiles are uniformly distributed inside cells. In the second distribution, mobiles are concentrated near to base stations while in the third distribution, mobiles are concentrated in cell borders (figure 2). The aim of using different types of mobile distribution is to show that crossed slots are suited in some situations where intermobile interference are not very high.

To generate the three types of mobile distribution, we consider that each mobile is located at a side of an hexagon $E$ that have the same center of the hexagonal cell. Each side of the hexagon $E$ is at a distance of $\rho$ meters from the center. The probability distribution functions of the three distributions are respectively: $1 / A, \sqrt{3} . R /(4 . \rho . A)$ and $\sqrt{3} . \rho / A$, where $A$ is the hexagonal cell surface and $R$ is the cell radius (figure 3 ).

Indexes $i$ and $k$ always refer to mobiles. Index $j$ refers to the base station that serves the studied mobile while index $l$ refers to a neighboring cell (fig. 1). The set of base station indexes is denoted $\Pi=\{1, \cdots, M\}$ and the set of mobiles connected to the base station $j$ during slot $n$ is denoted $S_{j, n}$.

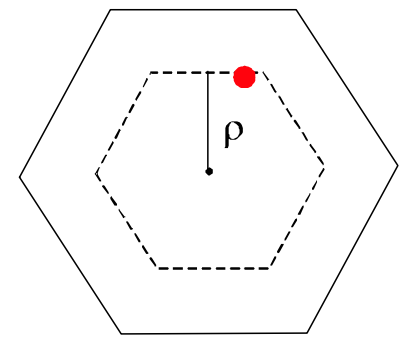

Fig. 3. The hexagon affected to the studied mobile and the distance $\rho$

A base station $j$ transmits a total power $P_{\mathrm{T}, j, n}$ during time slot $n$. This power is distributed between the mobiles of cell $j$ such that every mobile $i$ receives:

$$
C_{\mathrm{M}, i, n}=G_{i, j} \alpha_{i, n} P_{\mathrm{T}, j, n},
$$

where $\alpha_{i, n}$ is the portion of $P_{\mathrm{T}, j, n}$ dedicated to mobile $i$ during time slot $n$ and $G_{i, j}$ is the path gain between mobile $i$ and its server $j$.

The normalized path gain $Z_{i, l}$ between mobile $i$ and a neighboring base station $l$ is defined by:

$$
Z_{i, l}=\frac{G_{i, l}}{G_{i, j}} .
$$

If the $C I R$-balanced power control [8][9] is applied in a cell $j$, all communication links must be received with the same $C I R$. Moreover, all communication links are interfered by the same intercell-interference in uplinkcells. Therefore, all mobiles of an uplink-cell $j$ must be received with the same power $C_{\mathrm{B}, j, n}$ by their server. The received power is:

$$
G_{i, j} P_{\mathrm{M}, i, n}=C_{\mathrm{B}, j, n} \quad \forall i \in S_{j, n},
$$

where $P_{\mathrm{M}, i, n}$ is the power transmitted by mobile $i$. The value of $C_{\mathrm{B}, j, n}$ is not the same in all cells and is fixed by the power control scheme.

\section{Simplified OPTIMUM POWER CONTROL}

We suppose that the time slot allocation is done and we analyze the optimum power control in a crossed time slot. Uplink and downlink time slots are special cases of crossed time slots. In a crossed slot $n$, let $\Pi_{d, n}$ and $\Pi_{u, n}$ be the sets of downlink-cells and uplink-cells respectively. Our objective is to find the highest $C I R$ that can be achieved by all communication links of the system. This $C I R$ is reached when the optimum power control is used during each time slot independently. Therefore, only one time slot is studied.

In downlink-cells, the power control scheme is applied to the total power transmitted by base stations instead of specific power to each mobile. In uplink-cells, the power control scheme is applied to the received power in base stations. In crossed time slots, each type of link is studied independently; equations obtained in both links are combined to form one linear system. 
Let $\gamma_{n}$ be the $C I R$ that can be reached by all active mobiles during a time slot $n$, hence:

$$
\Gamma_{i, j, n} \geq \gamma_{n} \quad \forall i \in S_{j, n}, j \in \Pi,
$$

where $\Gamma_{i, j, n}$ is the estimated $C I R$ of mobile $i$.

\section{A. Downlink-cells}

The path gain between two mobiles $i$ and $k$ is denoted $G_{k, i}$. From equation (4), the power transmitted by mobile $k$ of cell $l$ is $C_{\mathrm{B}, l, n} / G_{k, l}$. The $C I R$ of a mobile $i$ in a downlink-cell $j$ is given by:

$$
\Gamma_{i, j, n}=\frac{\alpha_{i, n} G_{i, j} P_{\mathrm{T}, j, n}}{I_{\mathrm{d}, i, n}}
$$

where $I_{\mathrm{d}, i, n}$ is the total interference power received by mobile $i$ during time slot $n$ :

$$
\begin{aligned}
I_{\mathrm{d}, i, n} & =\underbrace{\beta_{d} G_{i, j}\left(1-\alpha_{i, n}\right) P_{\mathrm{T}, j, n}}_{\text {IntraI }}+\underbrace{\sum_{l \in \Pi_{d, n}-\{j\}} G_{i, l} P_{\mathrm{T}, l, n}}_{\text {InterID }} \\
& +\underbrace{\sum_{l \in \Pi_{u, n}}\left(\sum_{k \in S_{l, n}} \frac{G_{k, i}}{G_{k, l}}\right) C_{\mathrm{B}, l, n},}_{\text {Inter IU }}
\end{aligned}
$$

where IntraI is the intracell-interference power, Inter ID is the intercell-interference power received from neighboring base stations active in downlink and InterIU is the intercell-interference power received from all the neighboring mobiles active in the uplink.

By substituting the value of $\Gamma_{i, j, n}$ from (6) in (5) and using (3), we obtain:

$$
\begin{aligned}
\frac{\alpha_{i, n}}{\gamma_{n}} P_{\mathrm{T}, j, n} & \geq \beta_{d}\left(1-\alpha_{i, n}\right) P_{\mathrm{T}, j, n}+\sum_{l \in \Pi_{d, n}-\{j\}} Z_{i, l} P_{\mathrm{T}, l, n} \\
& +\sum_{l \in \Pi_{u, n}}\left(\sum_{k \in S_{l, n}} \frac{G_{k, i}}{G_{i, j} G_{k, l}}\right) C_{\mathrm{B}, l, n}
\end{aligned}
$$

By making the sum over all mobiles of cell $j$ and adding $P_{\mathrm{T}, j, n}$ to both sides of the last inequality, we obtain:

$$
\frac{1+\gamma_{n}}{\gamma_{n}} P_{\mathrm{T}, j, n} \geq P_{\mathrm{T}, j, n}+\Im_{\mathrm{d}, j, n}
$$

where $\Im_{\mathrm{d}, j, n}$ is given by:

$$
\begin{aligned}
\Im_{\mathrm{d}, j, n} & =\beta_{d}\left(N_{j, n}-1\right) P_{\mathrm{T}, j, n} \\
& +\sum_{l \in \Pi_{d, n}-\{j\}}\left(\sum_{i \in S_{j, n}} Z_{i, l} P_{\mathrm{T}, l, n}\right) \\
& +\sum_{l \in \Pi_{u, n}}\left(\sum_{i \in S_{j, n}} \sum_{k \in S_{l, n}} \frac{G_{k, i}}{G_{i, l} G_{k, l}}\right) C_{\mathrm{B}, l, n} .
\end{aligned}
$$

where $N_{j, n}$ is the number of active mobiles in cell $j$ during slot $n$.

If the intracell and intercell $C I R$-balanced power control is used, the set of relations (9) in all cells gives the same achievable $C I R$ as in [10] which is the maximum achievable $C I R$, because there is only one solution for (5) [9].

\section{B. Uplink-cells}

The $C I R$ of a mobile $i$ in an uplink-cell $j$ is:

$$
\Gamma_{i, j, n}=\frac{C_{\mathrm{B}, j, n}}{I_{\mathrm{u}, j, n}},
$$

where $I_{\mathrm{u}, j, n}$ is the total interference power corresponding to mobile $i$ during time slot $n$. This interference power is the same for all mobiles of cell $j$ :

$$
\begin{aligned}
I_{\mathrm{u}, j, n} & =\underbrace{\beta_{u}\left(N_{j, n}-1\right) C_{\mathrm{B}, j, n}}_{\text {IntraI }}+\underbrace{\sum_{l \in \Pi_{d, n}} G_{j, l} P_{\mathrm{T}, l, n}}_{\text {InterID }} \\
& +\underbrace{\sum_{l \in \Pi_{u, n}-\{j\}}\left(\sum_{k \in S_{l, n}} Z_{k, j} C_{\mathrm{B}, l, n}\right)}_{\text {InterIU }} .
\end{aligned}
$$

By substituting the value of $\Gamma_{i, j, n}$ from (11) in (5) and adding $C_{\mathrm{B}, j, n}$ to both sides of the last inequality we obtain:

$$
\frac{1+\gamma_{n}}{\gamma_{n}} C_{\mathrm{B}, j, n} \geq C_{\mathrm{B}, j, n}+I_{\mathrm{u}, j, n}
$$

\section{Generic model}

By combining equations (9) for all downlink-cells and (13) for all uplink-cells, we obtain a linear system which can be represented by the following matrix inequality:

$$
\mathbf{Z}^{(n)} \mathbf{R}_{n} \leq \frac{1+\gamma_{n}}{\gamma_{n}} \mathbf{R}_{n}
$$

where $\mathbf{R}_{n}=\left[R_{j, n}\right]$ is the $M \times 1$ vector with elements:

$$
R_{j, n}= \begin{cases}P_{\mathrm{T}, j, n} & \forall j \in \Pi_{d, n} \\ C_{\mathrm{B}, j, n} & \forall j \in \Pi_{u, n}\end{cases}
$$

$\mathbf{Z}^{(n)}$ is an $M \times M$ non negative matrix with elements:

$$
Z_{j, l}^{(n)}= \begin{cases}\sum_{i \in S_{j, n}} Z_{i, l} & \forall j, l \in \Pi_{d, n}, j \neq l \\ 1+\beta_{d}\left(N_{j, n}-1\right) & \forall j, l \in \Pi_{d, n}, j=l \\ \sum_{i \in S_{j, n}} \sum_{k \in S_{l, n}} \frac{G_{k, i}}{G_{i, j} G_{k, l}} & \forall j \in \Pi_{d, n}, l \in \Pi_{u, n} \\ G_{j, l} & \forall j \in \Pi_{u, n}, l \in \Pi_{d, n} \\ 1+\beta_{u}\left(N_{j, n}-1\right) & \forall j, l \in \Pi_{u, n}, j=l \\ \sum_{k \in S_{l, n}} Z_{k, j} & \forall j, l \in \Pi_{u, n}, j \neq l\end{cases}
$$

It must be noted that vector $\mathbf{R}_{n}$ includes the total base station transmitted powers in downlink-cells and the base station received powers in uplink-cells. From the Perron Frobenius theorem [11], the maximum value $\gamma_{n}^{*}$ of $\gamma_{n}$ that can be reached during a time slot $n$ with positive powers is obtained when the matrix inequality (14) became an equality:

$$
\mathbf{Z}^{(n)} \mathbf{R}_{n}^{*}=\frac{1+\gamma_{n}^{*}}{\gamma_{n}^{*}} \mathbf{R}_{n}^{*}
$$

and the value of $\gamma_{n}^{*}$ is given by the following equation:

$$
\gamma_{n}^{*}=\frac{1}{\lambda_{n}^{*}-1}
$$


where $\lambda_{n}^{*}$ is the largest real eigenvalue of the matrix $\mathbf{Z}^{(n)}$ and $\mathbf{R}_{n}^{*}$ is the corresponding eigen vector.

\section{System PERFormance AND Simulations RESULTS}

The maximum achievable $C I R$ is estimated in crossed and uplink slots using equation (18). The impact of the cell load and the impact of the asymmetry rate are evaluated for 1000 samples. Simulations results have shown that uplink slots have always higher average maximum achievable $C I R$ than crossed slots for all mobile distributions and for all asymmetry rates. In figure 4 , the crossed slot capacity is plotted as a function of the asymmetry rate for the three types of mobile distribution. The horizontal lines represent the uplink slot capacity, which are independent from the asymmetry rate. The slot capacity $C_{n}$ is the total maximum number of active mobiles that can be served during slot $n$ with a $C I R$ threshold $\gamma_{t h}$ :

$$
\begin{gathered}
C_{n}=\max \left(\sum_{j \in \Pi} \operatorname{card}\left(S_{j, n}\right)\right) / \\
\Gamma_{i, j, n} \geq \gamma_{t h} \forall i \in \bigcup_{j \in \Pi} S_{j, n}
\end{gathered}
$$

where card $(S)$ is the cardinality of the set $S$.

As it is expected, the slot capacity is at its highest level when the second distribution is used while it is at its lowest level when the third distribution is used; In the third distribution, the presence of a high number of active mobiles in uplink in some cells and in downlink in other cells at the cell borders increases the probability of high inter-mobile interference. Moreover, mobiles at the cell borders need more power than other mobiles to achieve $\gamma_{t h}$. Therefore, the third distribution has the lowest achievable $C I R$ while the second distribution has the highest one. Moreover, the crossed slot capacity is at its lowest level when the asymmetry rate is 0.5 . At this rate, the number of active mobiles in uplink and in downlink is at its maximum, and thus the probability that high inter-mobiles and inter-base stations interference affect the system, is at its maximum. The crossed slot capacity is higher when $a_{r}=1 / 10$ than when $a_{r}=9 / 10$, because $\beta_{u}>\beta_{d}$, and thus the intracell interference impact is higher in uplink than in downlink. Thus, when the number of uplink cells is higher than the number of downlink cells, the intracell interference impact is increased.

In figure 5, the probability density function (pdf) is plotted for the three distributions in uplink and crossed slots. The plotted figures correspond to a cell load of 6 codes/cell/slot and an asymmetry rate of $1 / 10$. As in figure 4 , the average maximum achievable $C I R$ is higher when the uplink is used and when mobiles are distributed using the second distribution. Furthermore, the range of pdf is very large in crossed slots while it is very narrow in uplink slots. This result is confirmed also when the confidence interval is evaluated; the range of confidence

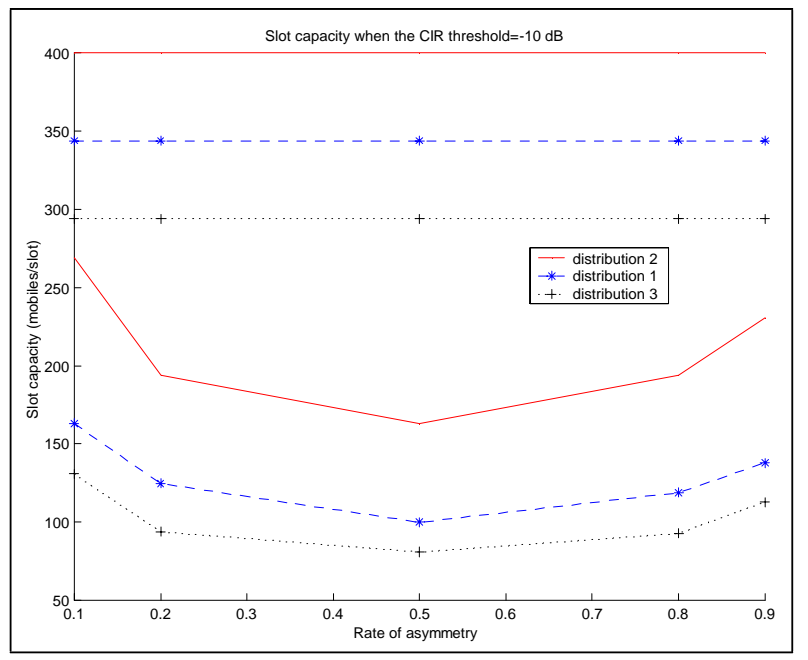

Fig. 4. The slot capacity for $\gamma_{t h}=-10 \mathrm{~dB}$

interval is higher in crossed slots than in uplink slots. The high variation in the maximum achievable $C I R$ in crossed slots is due to the high number of parameters affecting the path gain matrix. In uplink slots, only path gains between mobiles and base stations has an impact on the path gain matrix. In crossed slots, new type of path gains (i.e. path gains between mobiles and path gains between base stations) appear. The path gains between mobiles are highly dependent on the mobile positions. Hence, the path gain matrix in crossed slots may change drastically dependent on the mobile positions.

Though the mean of the maximum achievable $C I R$ is higher in uplink than in crossed slots, the maximum achievable $C I R$ in crossed slots is higher than the value in uplink slots for some mobile samples, especially when the second distribution is used. It must be noted that the maximum achievable $C I R$ can reach $-2.63 \mathrm{~dB}$ in crossed slots when the second distribution is used while the maximum value in uplink slot is $-3.5 \mathrm{~dB}$ for the same distribution.

The average difference between the maximum achievable $C I R$ in crossed and uplink slots is plotted in figure 6 with the confidence interval for three values of the asymmetry rate using the first distribution. The results show that the performance in crossed slots become closer to the performance of uplink slots for very high or very low values of $a_{r}$ in particular when $a_{r}$ is at its maximum where the slot become almost a pure downlink slot; for very high value of $a_{r}$ (resp. very low value of $a_{r}$ ), the number of uplink (resp. downlink) cells is very low and the probability of having high inter-mobile or inter-base station interference is very low.

Due to the high range of confidence interval, we can note also that the maximum achievable $C I R$ in crossed slots can be higher than its value in uplink slots in some cases (e.g. the difference can reach $5 \mathrm{~dB}$ ). However, the 


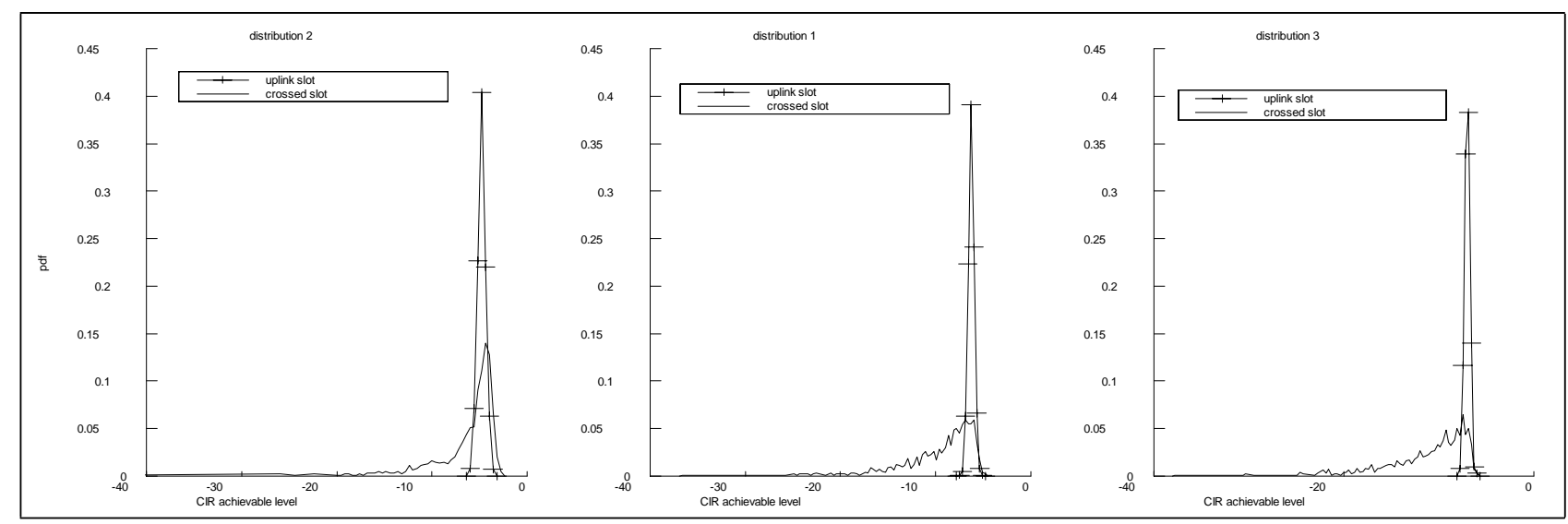

Fig. 5. The pdf of the maximum achievable CIR for uplink and crossed slots

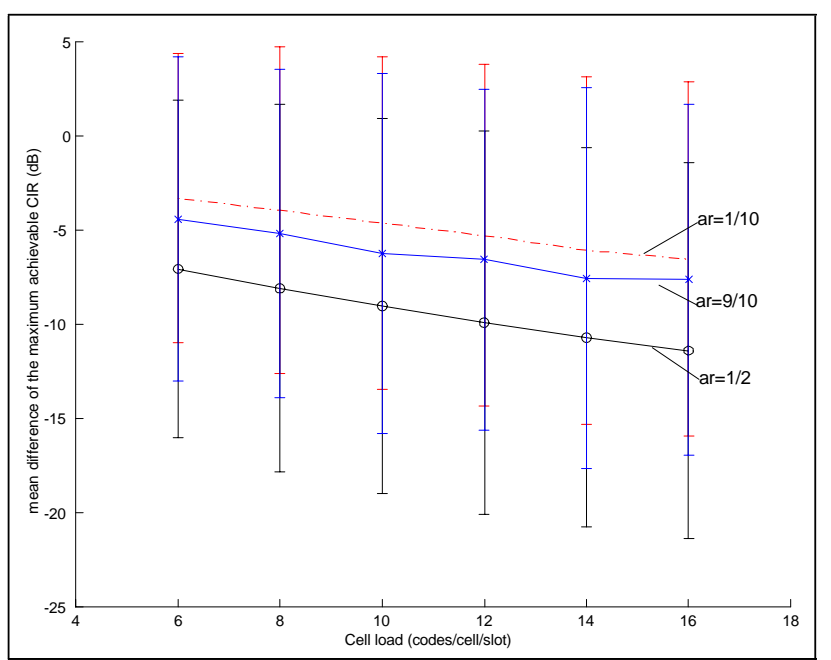

Fig. 6. The mean difference between the maximum achievable $C I R$ in uplink and crossed slots

performance in uplink is better in most cases and the enhancement in the uplink case can reaches $20 \mathrm{~dB}$ for high loads. The high range of confidence interval is due to the high variation of the maximum achievable $C I R$ in crossed slots.

\section{CONCLUSIONS}

In this paper, we have studied the performance of TDCDMA systems during crossed slots by using a simplified optimum power control and by considering the uplink slot as a reference. The impact of different mobile distributions inside cells, the cell load and the rate of asymmetry on the system performance during crossed slots were investigated. Simulation results has shown that in some distributions where mobiles are concentrated near to base stations, the system performance may be better during crossed slots than during uplink slots. However, the system performance in crossed slots varies in a large range depending on the path gain distributions. Therefore, a dramatic degradation may appear when high mobile-mobile interference are present though that some enhacements may appear in other cases. Therefore, crossed slot must be allocated very carefully for a low number of mobiles and intelligent slot allocation technique must be used to conserve the flexibility of the TD-CDMA technique without increasing the outage probability. This trade-off may be achieved by using crossed slots for cells where the mobile distribution is concentrated near to base stations and using some techniques to avoid high interference.

\section{REFERENCES}

[1] D. G. Jeong and W. S. Jeon, "Time slot allocation in cdma/tdd systems for mobile multimedia services," IEEE Communications Letters, vol. 4, no. 2, pp. $59-61,2000$.

[2] H. Holma, S. Heikkinen, O.-A. Lehtinen, and A. Toskala, "Interference considerations for the time division duplex mode of the UMTS terrestrial radio access," IEEE Journal on Selected Areas in communications, vol. 18, no. 8, 2000.

[3] J. Nasreddine and X. Lagrange, "Power control and slot allocation in a TD-CDMA system," in Proc.of the 55th IEEE Vehicular Technology Conference, 2002, 2002.

[4] J. Nasreddine and X. Lagrange, "Comparison of different slot allocation schemes in a TD-CDMA TDD system," in Proc.of the second IEEE Workshop on Applications and Services in Wireless Networks, 2002

[5] S. Verdu, Multiuser detection. Cambridge University Press, 1998.

[6] Siemens AG, "TDD UE-UE interference simulations," TSG-RAN Working Group 4 (Radio meeting n26), February 2003.

[7] J. Nasreddine and X. Lagrange, "Time slot allocation based on path gain division scheme for TD-CDMA TDD systems," in Proc.of the 57th IEEE Semiannual Vehicular Technology Conference, 2003, vol. 2 , pp. $1410-1414,2003$.

[8] J. M. Aein, "Power balancing in systems employing frequency reuse," COMSAT tech. rev., vol. 3, no. 2, 1973.

[9] J. Zander, "Performance of optimum transmitter power control in cellular radio systems," IEEE Transactions on Vehicular Technology, vol. 41, no. 1, pp. $57-62,1992$.

[10] $\mathrm{Q} . \mathrm{Wu}$, "Performance of optimum transmitter power control in CDMA cellular mobile systems," IEEE Transactions on Vehicular Technology, vol. 48, no. 2, pp. $571-575,1999$.

[11] F. R. Gantmacher, The theroy of matrices, vol. 2. New York: Chelsea publishing company, 1971. 